\title{
Unusual complications of intermittent self-catheterisation in spinal cord injury patients
}

\author{
S Vaidyanathan, KR Krishnan, BM Soni and MH Fraser \\ Regional Spinal Injuries Centre, District General Hospital, Southport, Merseyside PR8 6PN, UK
}

Three unusual complications of intermittent self-catheterisation in spinal cord injury patients observed over a period of 30 months are described. (1) Two patients performing intermittent catheterisation with a PVC catheter requiring the use of lignocaine gel to numb the urethra, developed allergic reaction in the form of swelling and erythematous lesions around the external urethral meatus. This rare complication was overcome by switching over to the use of a Lofric catheter instead of a PVC cetheter for intermittent catheterisation. (2) Urethral bleeding was observed on three occasions in male paraplegic patients performing intermittent self-catheterisation who had allowed the bladder to become over-distended. Bladder emptying therefore, took nearly $10 \mathrm{~min}$, by which time the Lofric catheter became too sticky in the urethra and required undue force for withdrawal, resulting in trauma to the adherent urethral mucosa and urethral bleeding. By adopting the policy of catheterisation at regular intervals and not allowing the bladder to become distended beyond $450 \mathrm{ml}$ of urine, this complication was never seen again either in these three patients or in any other patient using Lofric catheter for intermittent urethral catheterisation. (3) Failure to drain urine after urethral catheterisation resulting in a panic reaction was observed in a 45 year old woman practising intermittent catheterisation. This linique complication happened because there were no eye holes on the Nelaton catheter.

Keywords: intermittent catheterisation; spinal cord injury; urethra

\section{Introduction}

Intermittent catheterisation with the Lofric catheter has been recommended for use in spinal cord injury patients as it was associated with a significantly lesser degree of urethral inflammatory response when compared to the use of a PVC catheter. ${ }^{1}$ The reported complications associated with the use of a PVC catheter for intermittent catheterisation (size 12$14 \mathrm{Fr}$., vaseline lubricant, and the same catheter being used for 1 week), were (1) symptomatic lower urinary tract infection in $28 \%$; (2) epididymitis in $10 \%$; and (3) urethral stricture in $5.3 \%$ of cases. ${ }^{2}$ With the protocol of using disposable Lofric catheters of sizes 8,10 or $12 \mathrm{Fr}$. (with preference for the size $10 \mathrm{Fr}$. catheter) and sterile Normasol sachet for intermittent catheterisation, we have eliminated by and large, (1) the infective complications of intermittent catheterisation and (2) catheter-induced traumatic lesions of the urethra amongst nearly one hundred spinal cord injury patients (both adults as well as children) practising intermittent urethral catheterisation for management of neuropathic bladder. Herein, we describe three unusual complications of intermittent catheterisation observed

Correspondence: Dr S Vaidyanathan, Regional Spinal Injuries Centre, District General Hospital, Town Lane, Kew, Southport, Merseyside PR8 6PN, UK over a period of 30 months, which have not received adequate attention in the literature and therefore, the health professionals caring for the spinal cord injury patients are unaware of these potential complications, albeit they occur very rarely.

\section{Case reports}

Cases 1 and 2: allergic reaction to lignocaine gel A 34 year old woman with a neuropathic bladder because of sacral agenesis was performing intermittent catheterisation with a PVC catheter and was instilling lignocaine gel $\mathrm{BP} 2 \%$ (containing lignocaine hydrochloride BP $2 \% \mathrm{~m} / \mathrm{V}$ and chlorhexidine gluconate solution BP $0.25 \% \mathrm{v} / \mathrm{V}$ ) in the urethra to numb the unpleasant sensation which she was experiencing during catheterisation. After about 3 months, she developed erythematous lesions around the urethral orifice and over the labia. Initially, an infective pathology was suspected and she was prescribed ciprofloxacin after taking a specimen of urine for culture and swabs from the labia and vagina for bacteria, fungus, Trichomonas, and Chlamydia; microbiological tests on all these specimens subsequently proved to be negative. Allergy to lignocaine gel was then suspected as the area of erythematous lesions 
corresponded with the parts covered by spillage of the lignocaine gel as it was being squeezed into the urethral meatus. After discontinuing the use of lignocaine gel, the skin lesions disappeared promptly. She is now using a Lofric catheter, size $8 \mathrm{Fr}$. for intermittent catheterisation instead of the PVC catheter and there has been no problem since then.

A 46 year old male sustained traumatic paraplegia (T-8/9) in a road traffic accident in November 1993. Two months after the spinal trauma, he started performing self-catheterisation with a PVC catheter. He was using $2 \%$ lignocaine gel (containing lignocaine hydrochloride BP $2 \% \mathrm{~m} / \mathrm{V}$ and chlorhexidine gluconate solution BP $0.25 \% \mathrm{v} / \mathrm{V}$ ) to anaesthetise the urethra as his penis was painful while introducing the catheter. After a few days, he developed swelling of the penis, redness over the prepuce, glans penis, scrotum and medial aspect of the thighs in a patchy manner. These areas corresponded to the region which came in contact with the lignocaine gel. The clinical impression was allergic reaction to lignocaine gel. $\mathrm{He}$ switched over to the use of self-lubricating Lofric catheters for intermittent catheterisation as these catheters do not require any gel. The swelling of the penis and erythematous lesions regressed promptly. $\mathrm{He}$ did not feel sore in the penis while using the Lofric catheter.

\section{Cases 3, 4 and 5: over-distension of bladder and too} sticky Lofric catheter

Three adult male paraplegic patients had allowed the urinary bladder to become over-distended by either drinking too much fluid in a short spell of time (as during a drinking spree in patient number 3 ), or by performing catheterisation at infrequent intervals as in the case of latter two patients. When they performed catheterisation, there was three pints of urine in the bladder and it took nearly $10 \mathrm{~min}$ to empty the bladder. The technique of urethral catheterisation has not been faulty in any of them. Indeed, these patients have been performing self-catheterisation satisfactorily for at least 3 months. By the time the over-distended bladder was emptied, the outer surface of the prelubricated Lofric catheter became dry and sticky in the urethra. Considerable force was required for withdrawal of the sticky catheter and this resulted in trauma to the adherent urethral mucosa and hence, urethral bleeding. By adopting the policy of catheterisation at regular intervals and not allowing the bladder to get distended beyond $450 \mathrm{ml}$ of urine, this complication was never seen again either in these three patients or in any other patient using Lofric catheter for intermittent urethral catheterisation.

\section{Case 6: catheter without drainage holes}

A 45 year old woman has been practising intermittent catheterisation since August 1993 for the management of her neuropathic bladder, the cause being a large L5/
S central disc protrusion, for which a laminectomy of L5 and excision of L5-S1 disc were performed on 0707-1993. She had been using size 12 Fr. Nelaton, single use, prelubricated, female, catheters for the intermittent self-catheterisation, which was performed regularly at 4-5 hourly intervals and has been doing very well but for the recent episode. During a routine procedure of self-catheterisation, she passed a disposable catheter without any difficulty through the urethral opening. The catheter which appeared to have been introduced into the urethra to a reasonable length failed to drain urine. She could feel her bladder being full with urine; and thus expected the catheter to drain urine. But surprisingly, not a drop of urine was drained through the catheter, and she was panic-stricken. She removed the catheter. There was no urethral bleeding. She was confident that she had introduced the catheter into the urethra and not into the vagina. She again catheterised herself with the same catheter making sure that the catheter was introduced into the right passage. Even then, the catheter did not drain urine. She performed catheterisation using another catheter, and this time she drained about $300 \mathrm{ml}$ of clear urine. The mystery was solved when she closely examined the catheter with which she could not earlier drain urine from the bladder. There were no eye holes on the Nelaton catheter; it was a tube without drainage holes.

\section{Discussion}

A problem worth mentioning, although seen very rarely with the use of the Lofric catheter for intermittent catheterisation, is urethral bleeding; this happens because of the catheter becoming sticky in the urethra and requiring undue force for withdrawal, a manoeuvre which results in trauma to the adherent urethral mucosa and hence, urethral bleeding. Normally to prevent the surface from drying out, sodium chloride is incorporated on the surface of the Lofric catheter. The risk of the occurrence of this rare complication increases if patients reuse the catheter, as the amount of sodium chloride will be reduced, resulting in a decreased osmolality and thus increasing the risk of stickiness. Hence, we advise our patients not to reuse the Lofric catheter and to perform catheterisation at regular intervals.

When a catheter introduced to sufficient length in the urethra fails to drain urine, the possibilities are as follows:

(1) The catheter has become coiled in the urethra, a situation commonly encountered in male spinal cord injury patients in whom the Foley balloon had been earlier inflated in the prostatomembranous urethra thus producing a pseudocavity into which the catheter becomes coiled, instead of entering the bladder neck and the cavity of the bladder. We have also observed a similar situation in female spinal cord injury patients who have developed a capacious urethra (due to prolonged indwelling catheter drainage 
and frequent extrusion of the Foley balloon fully inflated), and who have subsequently undergone a Stamey-Gittes bladder neck suspension operation. The bladder neck suspension creates a 'shelf' across the bladder neck and thus produces resistance to the free passage of a catheter introduced per urethra; the catheter may then tend to coil inside the capacious urethra.

(2) The catheter has been introduced into a false passage in the urethra. This can happen only in male spinal cord injury patients, usually in those with urethral sphincter spasm. In female patients, the catheter may be inserted inadvertently into the vagina instead of the urethra. Such a mishap occurs either in very young children or in women with abnormal location of the external urethral meatus, eg female hypospadias.

(3) The catheter has been introduced correctly into the urinary bladder but its openings are blocked with the lubricating jelly and therefore it does not drain urine. This occurs only with those catheters requiring the use of a lubricant (eg K-Y lubricating jelly) and we have not encountered this problem while using a prelubricated catheter eg Lofric catheter.

(4) If the catheterisation had produced urethral trauma, the catheter might become blocked with a blood clot. There would be bleeding per urethra which provides a clue to the blockage of the lumen with blood clot(s) and the resultant failure to drain urine.

(5) Very rarely, a catheter may not have holes in its proximal end for the drainage of urine, as was the case in the patient reported here. This is due to a manufacturing defect and may occur very uncommonly with any type of catheter, be it PVC catheter, or prelubricated catheters, or Foley catheter. Alternatively, a 'catheter' may not have a drainage lumen if it was a urethral dilator, a soft tube being designed for use by patients, not for urinary drainage, but for selfdilatation of urethral meatus or urethral stricture. It is possible that inadvertently, a urethral dilator might have been placed instead of a catheter in a given carton and if a patient catheterises with a urethral dilator instead of a catheter, no urine will be drained through the tube. We had not considered such a manufacturing defect in the catheter or a misplaced urethral dilator instead of a catheter and, therefore, we had failed to appraise the patient of these rare possibilities; hence the patient panicked when the catheter did not drain urine.
After encountering these uncommon complications of intermittent catheterisation, we formulated a checklist for the spinal cord injury patients and health professionals caring for them to follow when any particular problem is encountered during intermittent catheterisation:

(1) While using the PVC catheter and lignocaine gel, if swelling and redness around the urethral meatus and adjacent areas is noticed, an allergic reaction to any of the constituents of the gel is a possibility. Preferably, the patient may switch over to the use of Lofric catheter which does not require the use of any gel.

(2) While performing intermittent catheterisation, the bladder should not be allowed to become overdistended. If a person drinks excessive amounts of fluids, more frequent catheterisation is indicated.

(3) The catheter should not be forcibly introduced into, or withdrawn from the urethra.

(4) If a prelubricated catheter (eg Lofric catheter) becomes sticky in the urethra, its outer surface should be moistened again by gently introducing sterile normal saline into the urethra.

(5) A new, sterile Lofric catheter should be used for each catheterisation as Lofric catheter is a 'single use' only item (as indicated in British National Formulary, number 31, March 1966, page 623).

(6) Medical attention should be sought immediately if a catheter, introduced gently into the urethra to its full length does not drain urine, or if there is bleeding while introducing the catheter, or at the end of catheterisation.

Hopefully, by following these guidelines, spinal cord injury patients on an intermittent catheterisation regime, will be able to avoid any untoward incident related to urethral catheterisation, and the level of awareness regarding the potential complications of intermittent catheterisation will be raised among the patients and the health professionals caring for the spinal cord injury patients.

\section{References}

1 Vaidyanathan S, Soni BM, Dundas S, Krishnan KR. Urethral cytology in spinal cord injury patients performing intermittent catheterisation. Paraplegia 1994; 32: 493-500.

2 Perrouin-Verbe B et al. Clean intermittent catheterisation from the acute period in spinal cord injury patients. Long term evaluation of urethral and genital tolerance. Paraplegia 1995; 33: $619-624$ 